\title{
LA VALORACIÓN DE LA DOCENCIA UNIVERSITARIA EN FUNCIÓN DEL SEXO Y DEL CENTRO DE LOS ESTUDIANTES ENCUESTADOS
}

\author{
APPRAISAL OF UNIVERSITY TEACHING ACCORDING TO THE SEX AND \\ INSTITUTION OF STUDENTS SURVEYED
}

\author{
Juan Ruiz Carrascosa* y David Molero López-Barajas**
}

Universidad de Jaén

\begin{abstract}
RESUMEN
En este artículo se presenta un estudio sobre la valoración de la docencia universitaria por parte del alumnado. A partir de un análisis factorial realizado en la elaboración del cuestionario podemos establecer la existencia de 4 factores de la evaluación de la docencia universitaria. Se analizan las dimensiones y variables más valoradas y se realiza un análisis de varianza para determinar si existen diferencias significativas, a nivel estadístico, entre las valoraciones realizadas en función del sexo de los encuestados y del centro al que pertenecen. Finalizamos el artículo con unas líneas futuras de trabajo al tener que adaptar la evaluación de la docencia universitaria a las directrices que marcan los estudios de grado y posgrado del Crédito Europeo.
\end{abstract}

Palabras Clave: Educación superior, opinión del alumnado, evaluación de la docencia, mejora de la enseñanza.

\begin{abstract}
This paper is about student's appraisal of university teaching. Factorial analysis of questionnaires shows four factors influencing the assessment of university teaching. The best valued variables and dimensions are analyzed, and analysis of variance is used for significant differences according to the sex or institution of the informants. The paper finally proposes initiatives for adaptation of university teaching to the guidelines of under and post-graduate studies under the European Credit System.
\end{abstract}

* Dr. Juan Ruiz Carrascosa. Profesor Titular de la Universidad de Jaén, Departamento de Pedagogía, Área MIDE. .Ex-Director del Secretariado de Metodología del Gabinete de Calidad de la Universidad de Jaén.ExCoordinador General del Proyecto de Evaluación del Profesorado de la Universidad de Jaén.

** Dr. David Molero López-Barajas. Profesor Asociado de Universidad de Jaén, Departamento de Pedagogía, Área MIDE. Ex-Colaborador del Proyecto de Evaluación del Profesorado de la Universidad de Jaén. Ex-Colaborador del Secretariado de Metodología del Gabinete de Calidad de la Universidad de Jaén 
Key words: Higher education, opinion of the student body, evaluation of the teaching, teaching improvement.

\section{Introducción}

A finales del Siglo XIX aparecen los primeros elementos sobre efectividad del profesorado a partir de la opinión del alumnado, Kratz en 1889 publica un trabajo sobre esta temática, el que es considerado como primer esfuerzo (Good y Mulryan, 1990). No es hasta los años veinte cuando comienza a estudiarse la calidad de la docencia en instituciones de educación superior. Los programas de evaluación por estudiantes se introdujeron en Harvard, la Universidad de Washington, la Universidad de Purdue y la Universidad de Texas.

Cook (1989) y Marsh (1987), señalan que la primera escala para estudiantes se publicó en 1927, fue la Purdue Rate Scale of Instruction de Remmers, aunque otros autores, como Good y Mulryan (1990), consideran que el primero en establecer un instrumento para medir la efectividad docente fue la escala de Eliot en 1915. Remmers et al. (1949) y Guthrie (1954) investigaron sobre los cuestionarios a los estudiantes durante los años treinta y cuarenta. Barr (1948) realizó un estudio en el que compiló 138 estudios sobre eficacia de la docencia escritos entre 1905 y 1948, y de Wolf (1990) reúne un conjunto de investigaciones sobre la evaluación de estudiantes de la efectividad docente.

Marsh (1987) resume los aspectos más relevantes de estos inicios sobre la investigación de la evaluación de los docentes por parte del alumnado. Establece que Remmers (1931; 1934) es el primero en reconocer que la fiabilidad de las encuestas a estudiantes puede estar basada en el acuerdo de diferentes alumnos sobre el mismo docente y que la fiabilidad de la respuesta media varía en el número de estudiantes de forma análoga entre la longitud del test y la fiabilidad del mismo.

Este mismo autor, Remmers (Remmers et al., 1949), publicó el primer análisis factorial de respuestas medias de los estudiantes, estableciendo diez rasgos, identificando dos de ellos como de orden superior, Empatía y Madurez Profesional (Creager, 1950). Drucker y Remmers (1950) determinaron que las encuestas a los alumnos diez años después de su graduación, en Purdue, estaban correlacionadas sustancialmente con las encuestas realizadas a los estudiantes actuales sobre aquellos profesores que habían tenido los dos grupos. Asimismo, había gran acuerdo sobre la relativa importancia que daban a los diez rasgos de la Escala Purdue. Remmers, en el primer estudio a gran escala multi-institucional (Remmers et al, 1949), correlacionó las respuestas de estudiantes de catorce colleges y universidades con una amplia variedad de variables sociodemográficas.

Los usos de estos instrumentos de medida van desde el ámbito estrictamente administrativo hasta el investigador, aunque son más frecuentes los primeros (González, 1997). Los primeros cuestionarios fueron utilizados por los administradores para obtener información utilizándose con propósitos sumativos de promoción, traslado y juicio de méritos; aunque también fue utilizado para el aumento del salario, medida impopular entre los docentes durante los años 80 (Good y Mulryan, 1990) lo cual hizo que se denominaran cuestionarios de mérito, por lo que pasaron a utilizarse y denominarse, mayoritariamente, como medida de eficacia docente.

Estos cuestionarios se empezaron a demandar en muchas instituciones y su uso variaba entre lo formativo, que incluía el desarrollo docente, a la utilidad sumativa que jugaba un 
papel importante en la toma de decisiones. Como establece Centra (1988; 1993), el profesorado tuvo varias razones para este movimiento: se empieza a economizar y muchas decisiones se empiezan a tomar por los contratantes, se focaliza la atención sobre los méritos ya que los presupuestos se recortan, aumentando los aspectos legales de la evaluación, convirtiéndose los cuestionarios en una forma de recoger información objetiva del profesorado.

El control y la evaluación del profesorado se convirtieron en aspectos importantes de la administración educativa. Hubo un aumento creciente de la burocratización de la educación pública que provocó el estudio de la efectividad docente y de desarrollo de estándares para el profesorado. El incremento de demandas de rendición de cuentas por parte del profesorado hizo posible el desarrollo de la evaluación de los docentes.

La creciente utilización de los cuestionarios para la evaluación del profesorado por parte de los alumnos en casi todas las universidades del mundo ha generado infinidad de estudios sobre éstos instrumentos de medida y sobre la docencia en la educación superior, publicándose un gran número de trabajos. En nuestro país, las primeras experiencias de evaluación del profesorado por encuestas al alumnado surgen en los años ochenta. En la actualidad la gran mayoría de las universidades españolas han puesto en marcha el proceso de evaluación de profesorado, centrando la estrategia de recogida de información, principalmente, en encuestas al alumnado. Los estudios son numerosos, siendo los primeros desde el punto de vista cronológico, entre otros, los siguientes: Aparicio, Tejedor y Sanmartín (1982) Jiménez (1985), Marsh, Tourón y Wheeler (1985), Tejedor (1985, 1986), Escudero (1986), Jornet et al (1987), Mateo (1987), Rodríguez Espinar (1987), Tejedor, Jato y Mínguez (1988), Escudero (1989), Miguel de (1989), García Ramos (1989).

Más adelante, ya en los años noventa, esta línea de investigación sigue teniendo productividad, como queda manifiesto en diversos trabajos (Abalde, et al 1995; Cajide, 1994; Cajide, Doval y Porto, 1996; García Ramos, 1997; García-Valcarcel, 1992; González Such et al, 1995; González, 1997; Jornet et al, 1996; Tejedor et al, 1995; Tejedor y García-Valcarcel, 1996).

La aprobación del I Plan Nacional de Evaluación de la Calidad de las Universidades (Real Decreto 1947/1995), y la del II Plan Nacional (Real Decreto 408/2001), regula los procesos de evaluación institucional en las universidades españolas, lo que ha favorecido la difusión del la evaluación de la calidad docente como parte de la evaluación de la enseñanza para avanzar en el proceso de evaluación institucional (Ruiz, 2000).

En los últimos años se han seguido realizando estudios en nuestro país (Apodaca y Grad, 2002; Escudero, 2002; García Ramos, 1999; Mateo, 2000; Molero, 2003; Molero, 2004a, 2004b; Molero y Ruiz, 2003; Muñoz, Rios y Abalde, 2002, 2003; Rodríguez Gómez, 2000; Tejedor, 2003; Tierno y Jiménez, 2003). La mayor parte de los trabajos citados están relacionados con evaluaciones de la docencia desarrolladas en nuestras universidades, aunque también aparecen investigaciones sobre las características métricas de los instrumentos de recogida de información o sobre los modelos de evaluación empleados.

A pesar de esta variedad de estudios, el Informe Global del Plan Nacional de Evaluación de la Calidad de las Universidades 1996-2000 (Consejo de Coordinación Universitaria, 2002, p. 37) establece que: "La evaluación de la actividad docente del profesorado es abordada de forma heterogénea por parte de las unidades evaluadas. Entre las debilidades señaladas por las titulaciones resaltan la ausencia de mecanismos de evaluación del profe- 
sorado, las deficiencias en los procedimientos de evaluación existentes y la escasa valoración que se realiza de la evaluación docente".

Por tanto, estudios como el que presentamos son necesarios para institucionalizar el proceso de evaluación de la docencia universitaria, creando cultura evaluativa sobre este aspecto en las universidades que aún no hayan desarrollado estos mecanismos y afianzar y reforzarlos en las instituciones en los que parecen estar más asentados.

\section{Problema de investigación}

El problema de investigación que nos planteamos aborda varias cuestiones. La primera de ellas es determinar si es posible conocer la opinión del alumnado de la Universidad de Jaén en las diferentes facultades y centros respecto a la docencia que imparte el profesorado. La segunda cuestión que nos planteamos en nuestra investigación, es determinar la existencia de diferencias significativas, a nivel estadístico, entre las valoraciones de la docencia efectuadas por hombres y por mujeres y entre el alumnado de los distintos centros o facultades.

\section{Objetivos}

Considerando el problema de investigación, planteado anteriormente, los objetivos del estudio, con carácter general, son los siguientes:

- Conocer y analizar la opinión del alumnado de la Universidad de Jaén sobre la docencia que se imparte en esta Universidad.

- Conocer la valoración de la docencia por parte del alumnado en cada una de las Facultades o Centros de nuestra Universidad.

- Determinar la existencia de diferencias significativas a nivel estadístico en las valoraciones de la docencia realizadas por el alumnado en función del sexo de los encuestados.

- Determinar la existencia de diferencias significativas a nivel estadístico entre las valoraciones realizadas por el alumnado en función de la Facultad o Centro al que pertenecen.

\section{Muestra}

La muestra del estudio responde a un muestreo incidental (Buendía, 1994, p. 94). Los sujetos que componen la muestra son los estudiantes de primer y segundo ciclo de la Universidad de Jaén que contestaron el Cuestionario de Evaluación de la Docencia Universitaria de la Universidad de Jaén durante el curso académico 2002/2003, siendo un total de $14052(n=14052)$.

La distribución de la muestra en función del sexo y de la Facultad o Centro al que pertenecen, aparece reflejada en la tabla 1 . El $51.06 \%$ de los encuestados son hombres y el $48.94 \%$ mujeres. En relación con la Facultad o Centro en donde cursan estudios, el 26.62\% 
son de la Escuela Politécnica Superior, el 22.12\% de la Facultad de Ciencias Sociales y Jurídicas, el 16.62\% de la Facultad de Ciencias Experimentales, el 15.52\% de la Escuela Universitaria Politécnica de Linares, el $10.10 \%$ de la Facultad de Humanidades y Ciencias de la Educación, 4.78\% de la Escuela Universitaria de Trabajo Social de Linares y el 4.20\% de la Escuela Universitaria de Enfermería.

TABLA 1: Distribución de la muestra.

\begin{tabular}{|c|c|c|c|}
\hline \multirow{2}{*}{ Facultad o Centro } & \multicolumn{2}{|c|}{ Sexo } & \multirow{2}{*}{ Total } \\
\hline & Hombre & Mujer & \\
\hline Escuela Politécnica Superior & 2946 & 796 & 3742 \\
\hline Facultad de Ciencias Sociales y Jurídicas & 1070 & 2039 & 3109 \\
\hline Facultad de Ciencias Experimentales & 871 & 1465 & 2336 \\
\hline E. U. Politécnica de Linares & 1688 & 494 & 2182 \\
\hline Facultad de Humanidades y C.C.E.E. & 413 & 1007 & 1420 \\
\hline E. U. de Trabajo Social de Linares & 83 & 589 & 672 \\
\hline Escuela Universitaria de Enfermería & 104 & 487 & 591 \\
\hline Total & 7175 & 6877 & 14052 \\
\hline
\end{tabular}

\section{Metodología}

En función del problema de investigación y los objetivos planteados, hemos optado por una metodología de tipo descriptivo y de relación, para procurar determinar la incidencia de las variables Sexo y Centro tras la valoración de la docencia universitaria por grupos constituidos por sujetos que presentan diferencias en las variables citadas. Dentro de los diferentes tipos de estudios descriptivos utilizamos los de tipo encuesta y como instrumento de recogida de información un cuestionario.

\section{Instrumento}

El instrumento de recogida de información empleado ha sido el Cuestionario de Evaluación de la Docencia Universitaria (véase anexo), el cual ha sido aplicado en diferentes ocasiones con resultados satisfactorios (Universidad de Jaén, 2002). El instrumento fue sometido a la comprobación del cumplimiento de sus características técnicas: validez y fiabilidad.

\section{Validez}

La validez de contenido se procuró a través de dos vías. La primera de ellas fue la revisión de la literatura especializada más relevante de la temática que nos ocupa (Abrami, Co- 
hen y d'Apollonia, 1990; Aleamoni, 1981; Apodaca y Grad, 2002; Centra, 1993, 2000; Doyle, 1983; García Ramos, 1999; Marsh, 1987; Marsh y Roche, 1992, 1993; Muñoz, Rios y Abalde, 2002; Tejedor, 1985, 1986, 2003; Wilson, 1999) y el estudio de las vías para la evaluación de la docencia universitaria empleadas en diferentes universidades españolas. La segunda de las vías de análisis de la validez de contenido se desarrolló por medio de una consulta a jueces expertos, que en nuestro caso fueron profesores del área de Métodos de Investigación y Diagnóstico en Educación de diferentes universidades españolas.

En relación con la validez de constructo, se realizó un análisis factorial con el fin de comprobar si podían hallarse factores consistentes. Para ello se realizó el análisis de los ítems del cuestionario que evalúan la docencia los cuales responden a una escala de contestación tipo Likert de 5 alternativas con el fin de delimitar los componentes principales y determinar la varianza total que éstos explican.

Para considerar si ese análisis es idóneo se ha contrastado a partir de diferentes medidas. El test de esfericidad de Bartlett ofreció un valor de 274797.7, siendo, el resultado de la medida de adecuación muestral de kaiser-Meyer-Olkin fue de 0.969 , lo que puede calificarse como excelente de cara a la realización del análisis factorial. Estos dos resultados corroboran la idoneidad de la matriz de correlaciones, por lo que el citado análisis era pertinente (Tabla 2).

El modelo de extracción de factores utilizado es el de Componentes Principales (MCP), ya que el objetivo es encontrar una serie de componentes que expliquen el máximo de varianza total de las variables originales. Hemos seleccionado el Modelo de Componentes Principales (MCP) ya que es, posiblemente, la técnica exploratoria más utilizada y aceptada en la investigación socioeducativa (Gavira, 2000, p. 42). Los cuatro factores incluidos en el modelo son capaces de explicar un $64.777 \%$ de la variabilidad total.

\section{Fiabilidad}

La fiabilidad de la prueba fue estimada a través del cálculo del coeficiente $\alpha$ (alfa de Cronbach), para determinar la consistencia interna, obteniéndose un coeficiente igual a 0.9631, con un nivel de confianza del 95\%, para el total de los ítems que evalúan la docencia universitaria.

En el Factor I: Interacción con el alumnado, el coeficiente de fiabilidad obtenido es igual a 0,9225; en el Factor II: Metodología, el coeficiente obtenido es igual a 0,9053; en el Factor III: Obligaciones Docentes-Evaluación, es igual a 0,8747; y en el Factor IV: Medios y recursos, el coeficiente es igual a 0,7783 .

\section{Análisis y valoración de los resultados}

\section{Estudio descriptivo}

La valoración de la docencia por el alumnado que forma la muestra no puede calificarse como negativa, si tenemos en cuenta que para el total de la escala la media se situó en 3,80; obteniéndose en los 25 ítems contemplados puntuaciones medias superiores al valor central 3. Si realizamos una caracterización del alumnado en función de sus puntuaciones totales, encontramos que solo un $14,2 \%$ de los estudiantes han obtenido valores inferiores a 3 , fren- 


\section{TABLA 2: Dimensiones factoriales del Cuestionario de Evaluación de la Docencia Universitaria.}

\begin{tabular}{|c|c|}
\hline \multicolumn{2}{|r|}{ Factor 1: Interacción con el alumnado } \\
\hline P.6. & El profesor tiene en cuenta la opinión del alumnado en la marcha de la asignatura. \\
\hline P.7. & El profesor muestra interés en que el alumno aprenda. \\
\hline P.8. & El profesor motivó y facilitó la participación del alumnado en clase. \\
\hline P.9. & Existe una buena relación entre el profesor y el alumno. \\
\hline P.10. & El profesor ha contribuido a que me guste la asignatura. \\
\hline P.11. & El profesor ha contribuido a que comprenda la importancia de la asignatura. \\
\hline \multicolumn{2}{|r|}{ Factor 2: Metodología } \\
\hline P.3. & El programa se define con claridad (metodología y contenidos). \\
\hline P.12. & El profesor imparte sus clases con claridad, organización y coherencia. \\
\hline P.13. & El profesor responde con exactitud y precisión a las preguntas que se le hacen. \\
\hline P.14. & El profesor domina la asignatura que imparte. \\
\hline P.15. & $\begin{array}{l}\text { La metodología de enseñanza utilizada resulta adecuada a las características del grupo y } \\
\text { de la asignatura. }\end{array}$ \\
\hline P.19. & Sus clases están bien preparadas. \\
\hline \multicolumn{2}{|r|}{ Factor 3: Obligaciones Docentes y Evaluación } \\
\hline P.1. & El profesor ha cumplido el horario de clases. \\
\hline P.2. & El profesor atiende correctamente al alumnado en las horas de tutoría. \\
\hline P.4. & El profesor anticipo los objetivos del curso y de cada tema. \\
\hline P.5. & $\begin{array}{l}\text { El método de evaluación del profesor ha sido conocido con suficiente antelación a la } \\
\text { fecha de examen. }\end{array}$ \\
\hline P.21. & El profesor ha cumplido el programa de la asignatura planteado al inicio del curso. \\
\hline P.22. & $\begin{array}{l}\text { Los exámenes / evaluaciones que se han realizado se ajustan a los objetivos y a los } \\
\text { contenidos trabajados en clase. }\end{array}$ \\
\hline P.23. & El profesor respeta los criterios de evaluación establecidos en la asignatura. \\
\hline P.24. & El sistema de evaluación de la asignatura permite revisión por parte del alumnado. \\
\hline \multicolumn{2}{|r|}{ Factor 4: Medios y Recursos } \\
\hline P.16. & $\begin{array}{l}\text { Los problemas, ejemplos o prácticas que plantea, están bien pensados para el contexto } \\
\text { de la asignatura. }\end{array}$ \\
\hline P.17. & El material técnico y de laboratorio necesario para esta asignatura es el apropiado. \\
\hline P.18. & La bibliografía y material didáctico recomendado resulta útil para preparar la asignatura. \\
\hline P.20. & Existe coordinación entre la parte teórica y práctica de la asignatura. \\
\hline
\end{tabular}


te al $83.8 \%$ restante que alcanza o supera ese nivel. Por lo tanto podemos calificar la valoración de la docencia universitaria basada en la opinión del alumnado de la muestra como moderadamente positiva, a continuación destacamos las puntuaciones más relevantes.

Como podemos apreciar en la tabla 3 , el ítem con una puntuación más baja es el número 10 , en el que se valora si el profesorado contribuye a que guste la asignatura que imparte, con una puntuación media igual a 3,41. Otros ítems con puntuaciones reducidas son el número 17, el material técnico y de laboratorio es el apropiado, y el ítem 11, el profesor ha contribuido a comprenda la importancia de la asignatura. Las puntuaciones medias de estas dos variables son de 3,50 y 3,55 respectivamente.

Entre las cuestiones más valoradas se encuentran la número 1, el profesor ha cumplido el horario de clase, y la número 14, el profesor domina la asignatura que imparte; con medias de 4,31 y 4,30, respectivamente. En el primero de estos ítems, el número 1, el 56.7\% de los estudiantes encuestados dan la máxima valoración a los docentes en esta variable.

$\mathrm{Si}$ analizamos las puntuaciones alcanzadas en cada una de las dimensiones (véase tabla 4) podremos obtener una visión más amplia sobre la valoración de la docencia por parte del alumnado.

TABLA 4: Estadísticos descriptivos, medias y desviaciones típicas globales y por dimensiones.

\begin{tabular}{|c|c|c|}
\hline Dimensiones & Media & Desv. Típ. \\
\hline 1. Interacción con el alumnado & 3,65 & ,9683 \\
\hline 2. Metodología & 3,89 & ,8363 \\
\hline 3. Obligaciones Docentes y Evaluación & 3,95 &, 7252 \\
\hline 4. Medios y Recursos & 3,72 &, 8346 \\
\hline Total de la Encuesta & 3,80 & ,7378 \\
\hline
\end{tabular}

La dimensión con una mayor puntuación media es la relacionada con las Obligaciones Docentes y Evaluación, la cual posee una puntuación media cercana a 4 puntos (media igual a 3.95), seguida de la segunda Metodología (media igual a 3.89), Medios y Recursos es la tercera más valorada (puntuación media de 3.72) y por último, la dimensión Interacción con el alumnado con una puntuación media igual a 3.65 .

\section{Análisis de la varianza}

Vamos a realizar dos análisis de la varianza (ANOVA) con la intención de conocer si existen diferencias significativas, a nivel estadístico, en las valoraciones realizadas por el alumnado en función del sexo y del centro de los encuestados.

En el primero de los análisis tomaremos como variable independiente el sexo de la muestra y como variables dependientes las valoraciones realizadas en cada una de las dimensiones y la valoración total de la encuesta. Los grupos de la variable independiente para este ANOVA son los siguientes: Grupo: 1 Hombre y Grupo 2: Mujer. 
TABLA 3: Estadísticos descriptivos, medias y desviaciones típicas alcanzadas en los ítems, con ordenación según criterio medias descendentes.

\begin{tabular}{|c|c|c|c|}
\hline & Ítems & Media & $\begin{array}{l}\text { Desv. } \\
\text { Típ. }\end{array}$ \\
\hline P.1 & El profesor ha cumplido el horario de clase & 4,31 & ,970 \\
\hline P.14 & El profesor domina la asignatura que imparte & 4,30 & ,914 \\
\hline P.2 & El profesor atiende correctamente al alumnado en las horas de tutoría & 4,10 & ,970 \\
\hline P.24 & El sistema de evaluación permite la revisión por parte del alumnado & 4,03 & ,977 \\
\hline P.19 & Sus clases están bien preparadas & 3,94 & 1,041 \\
\hline P.21 & El profesor ha cumplido el programa de la asignatura planteado & 3,93 & 1,031 \\
\hline P.23 & $\begin{array}{l}\text { El profesor respeta los criterios de evaluación establecidos en la } \\
\text { asignatura }\end{array}$ & 3,89 & ,941 \\
\hline P.7 & El profesor muestra interés en que el alumnado aprenda & 3,89 & 1,091 \\
\hline P.13 & El profesor responde con exactitud y precisión a las preguntas & 3,88 & 1,036 \\
\hline P.16 & Los problemas, ejemplos o prácticas que plantea, están bien pensados & 3,87 & 1,005 \\
\hline P.3 & El programa se define con claridad (metodología y contenidos) & 3,84 & 1,035 \\
\hline P.20 & Existe coordinación entre la parte teórica y la práctica de la asignatura & 3,84 & 1,106 \\
\hline P.5 & El método de evaluación ha sido conocido con suficiente antelación & 3,81 & 1,106 \\
\hline P. 25 & Estoy satisfecho respecto al profesor de la asignatura & 3,80 & 1,093 \\
\hline P.9 & Existe una buena relación entre el profesor y el alumnado & 3,79 & 1,101 \\
\hline P.22 & Los exámenes se ajustan a los objetivos y a los contenidos trabajados & 3,77 & 1,016 \\
\hline P.12 & El profesor imparte sus clases con claridad, organización y coherencia & 3,74 & 1,151 \\
\hline P.4 & El profesor anticipó los objetivos del curso y de cada tema & 3,74 & 1,049 \\
\hline P.6 & El profesor tiene en cuenta la opinión del alumnado & 3,68 & 1,121 \\
\hline P.15 & La metodología de enseñanza utilizada resulta adecuada a la asignatura & 3,65 & 1,054 \\
\hline P.18 & La bibliografía y material didáctico recomendado resulta útil & 3,64 & 1,051 \\
\hline P.8 & El profesor motivó y facilitó la participación del alumnado en clase & 3,62 & 1,174 \\
\hline P.11 & $\begin{array}{l}\text { El profesor ha contribuido a que comprenda la importancia de la } \\
\text { asignatura }\end{array}$ & 3,55 & 1,113 \\
\hline P.17 & El material técnico y de laboratorio es el apropiado & 3,50 & 1,179 \\
\hline P.10 & El profesor ha contribuido a que me guste la asignatura & 3,41 & 1,215 \\
\hline
\end{tabular}

Como podemos comprobar en la tabla 5 existen diferencias significativas, a nivel estadístico (nivel de confianza 95\%), entre las valoraciones realizadas por hombres y las rea- 
lizadas por mujeres en cada una de las dimensiones del cuestionario y en la valoración global de la docencia universitaria evaluada en este estudio; al ser los valores obtenidos en la $F$ experimental mayores al valor crítico de $F(F$ crítico $=3.84)$, en todos los casos.

TABLA 5: ANOVA (Sexo - Dimensiones y Valoración general).

\begin{tabular}{|c|c|c|c|c|c|c|}
\hline $\begin{array}{c}\text { Variables } \\
\text { Dependientes }\end{array}$ & \multicolumn{2}{|c|}{ Suma de cuadrados } & gl & $\begin{array}{c}\text { Media } \\
\text { cuadrática }\end{array}$ & $\mathbf{F}$ & Sig. \\
\hline \multirow{3}{*}{ Dimensión 1} & Inter-grupos & 65,443 & 1 & 65,443 & \multirow{3}{*}{70,245} & \multirow{3}{*}{, $000^{*}$} \\
\hline & Intra-grupos & 13088,637 & 14049 & ,932 & & \\
\hline & Total & 13154,080 & 14050 & & & \\
\hline \multirow{3}{*}{ Dimensión 2} & Inter-grupos & 85,801 & 1 & 85,801 & \multirow{3}{*}{124,025} & \multirow{3}{*}{, $000^{*}$} \\
\hline & Intra-grupos & 9719,899 & 14050 & ,692 & & \\
\hline & Total & 9805,701 & 14051 & & & \\
\hline \multirow{3}{*}{ Dimensión 3} & Inter-grupos & 95,128 & 1 & 95,128 & \multirow{3}{*}{183,834} & \multirow{3}{*}{, $000 *$} \\
\hline & Intra-grupos & 7269,907 & 14049 &, 517 & & \\
\hline & Total & 7365,035 & 14050 & & & \\
\hline \multirow{3}{*}{ Dimensión 4} & Inter-grupos & 228,968 & 1 & 228,968 & \multirow{3}{*}{337,469} & \multirow{3}{*}{, $000^{*}$} \\
\hline & Intra-grupos & 9518,484 & 14029 & ,678 & & \\
\hline & Total & 9747,453 & 14030 & & & \\
\hline \multirow{3}{*}{ Valoración Total } & Inter-grupos & 99,304 & 1 & 99,304 & \multirow{3}{*}{84,124} & \multirow{3}{*}{, $000^{*}$} \\
\hline & Intra-grupos & 16388,231 & 13883 & 1,180 & & \\
\hline & Total & 16487,535 & 13884 & & & \\
\hline
\end{tabular}

VI: Sexo (Grupo 1: Hombre, Grupo 2: Mujer).

VD: Dimensión 1, Dimensión 2, Dimensión 3, Dimensión 4, Valoración General.

(*) Diferencias significativas a nivel 0.05 .

En el segundo análisis de la varianza la variable independiente elegida es el Centro o Facultad a la que pertenecen los encuestados y como variables dependientes las valoraciones realizadas en cada una de las dimensiones y la valoración total de la encuesta. En importante definir claramente los grupos de esta variable independiente ya que son varios; Grupo1: Escuela Politécnica Superior de Jaén, Grupo 2: Escuela Universitaria Politécnica de Linares, Grupo 3: Escuela Universitaria de Trabajo Social, Grupo 4: Facultad de Ciencias Experimentales, Grupo 5: Facultad de Ciencias Sociales y Jurídicas, Grupo 6: Facultad de Humanidades y Ciencias de la Educación, Grupo 7: Escuela Universitaria de Enfermería.

Como podemos apreciar en la tabla 6 , existen diferencias significativas, a nivel estadístico (nivel de confianza 95\%), entre las valoraciones realizadas entre el alumnado de cada Centro o Facultad de la Universidad de Jaén en las dimensiones del cuestionario y en la va- 
loración global; al ser los valores obtenidos en la $F$ experimental mayores al valor crítico de $F(F$ crítico $=3.84)$. Pero es preciso realizar una prueba a posteriori o post hoc como la prueba de Scheffé, para determinar entre que grupos son los que se dan las diferencias significativas.

Tras el cálculo de esta prueba obtenemos que en la primera de las dimensiones (Interacción con el alumnado) existen diferencias significativas a nivel estadístico entre la Escuela Politécnica Superior y los demás centros o facultades, entre la E.U. Politécnica y la E.U. de Trabajo Social, entre la E.U. Politécnica y la Facultad de Ciencias Sociales y Jurídicas, entre E.U. Politécnica y la Facultad de Humanidades y C.C.E.E., entre la E.U. de Trabajo Social y la Facultad de Ciencia Experimentales, entre la E.U. de Trabajo Social y la Facultad de Humanidades y C.C.E.E., entre la Facultad de Ciencias Experimentales y la Facultad de Ciencias Sociales y Jurídicas, entre la Facultad de Ciencias Experimentales y la Facultad de Humanidades y C.C.E.E., entre la Facultad de Ciencias Sociales y Jurídicas y la Facultad de Humanidades y C.C.E.E., y finalmente, entre la Facultad de Humanidades y C.C.E.E. y la E.U. de Enfermería (Tabla 6).

En la segunda dimensión (Metodología) existen diferencias significativas, a nivel estadístico, entre la E. Politécnica Superior y la Facultad de Ciencias Experimentales, entre la E. Politécnica Superior y la Facultad de Ciencias Sociales y Jurídicas, entre la E. Politécnica Superior y la Facultad de Humanidades y C.C.E.E., entre la E. Politécnica Superior y la E.U. de Enfermería, entre E. U. Politécnica y la Facultad de Ciencias Sociales y Jurídicas, entre la E.U. Politécnica y la Facultad de Humanidades y C.C.E.E., entre la E.U. Politécnica y la E.U. de Enfermería, entre la E.U. de Trabajo Social y la Facultad de Ciencias Sociales y Jurídicas, entre la E.U. de Trabajo Social y la Facultad de Humanidades y C.C.E.E., entre la E.U. de Trabajo Social y la E.U. de Enfermería, entre la Facultad de Ciencias Experimentales y la Facultad de Ciencias Sociales y Jurídicas, entre la Facultad de Ciencias Experimentales y la Facultad de Humanidades y C.C.E.E., y por último entre la Facultad de Ciencias Experimentales y la E.U. de Enfermería.

En la tercera de las dimensiones (Obligaciones docentes y Evaluación) existen diferencias significativas a nivel estadístico entre la E. Politécnica Superior y la E.U. Politécnica, entre la E. Politécnica Superior y la Facultad de Ciencias Experimentales, entre la E. Politécnica Superior y la Facultad de Ciencias Sociales y Jurídicas, entre la E. Politécnica Superior y la Facultad de Humanidades y C.C.E.E., entre la E. Politécnica Superior y la E.U. de Enfermería, entre la E.U. Politécnica y la Facultad de Ciencias Sociales y Jurídicas, entre la E.U. Politécnica y la Facultad de Humanidades y C.C.E.E., entre la E.U. Politécnica y la E.U. de Enfermería, entre la E.U. de Trabajo Social y la Facultad de Ciencias Sociales y Jurídicas, entre la E.U. de Trabajo Social y la Facultad de Humanidades y C.C.E.E., entre la E.U. de Trabajo Social y la E.U. de Enfermería, entre la Facultad de Ciencias Experimentales y la Facultad de Ciencias Sociales y Jurídicas, entre la Facultad de Ciencias Experimentales y la Facultad de Humanidades y C.C.E.E., entre la Facultad de Ciencias Experimentales y la E.U. de Enfermería, y finalmente, entre la Facultad de Ciencias Sociales-Jurídicas y la Facultad de Humanidades y C.C.E.E.

Para la última de las dimensiones del cuestionario (Medios y Recursos) las diferencias entre los grupos son significativas a nivel estadístico entre la E. Politécnica Superior y la E.U. de Trabajo Social, la E. Politécnica Superior y la Facultad de Ciencias Experimentales, 
TABLA 6: ANOVA (Centro - Dimensiones y Valoración general).

\begin{tabular}{|c|c|c|c|c|c|c|}
\hline $\begin{array}{c}\text { Variables } \\
\text { Dependientes }\end{array}$ & \multicolumn{2}{|c|}{ Suma de cuadrados } & gl & $\begin{array}{c}\text { Media } \\
\text { cuadrática }\end{array}$ & $\mathbf{F}$ & Sig. \\
\hline \multirow{3}{*}{ Dimensión 1} & Inter-grupos & 369,207 & 6 & 61,535 & \multirow{3}{*}{67,470} & \multirow{3}{*}{, 000} \\
\hline & Intra-grupos & 12965,460 & 14216 & ,912 & & \\
\hline & Total & 13334,667 & 14222 & & & \\
\hline \multirow{3}{*}{ Dimensión 2} & Inter-grupos & 275,923 & 6 & 45,987 & \multirow{3}{*}{67,596} & \multirow{3}{*}{, 000} \\
\hline & Intra-grupos & 9672,179 & 14217 & ,680 & & \\
\hline & Total & 9948,103 & 14223 & & & \\
\hline \multirow{3}{*}{ Dimensión 3} & Inter-grupos & 196,006 & 6 & 32,668 & \multirow{3}{*}{63,749} & \multirow{3}{*}{, 000} \\
\hline & Intra-grupos & 7285,344 & 14217 &, 512 & & \\
\hline & Total & 7481,350 & 14223 & & & \\
\hline \multirow{3}{*}{ Dimensión 4} & Inter-grupos & 319,474 & 6 & 53,246 & \multirow{3}{*}{78,948} & \multirow{3}{*}{, 000} \\
\hline & Intra-grupos & 9574,981 & 14197 & ,674 & & \\
\hline & Total & 9894,454 & 14203 & & & \\
\hline \multirow{3}{*}{ Valoración Total } & Inter-grupos & 374,802 & 6 & 62,467 & \multirow{3}{*}{53,474} & \multirow{3}{*}{, 000} \\
\hline & Intra-grupos & 16407,066 & 14045 & 1,168 & & \\
\hline & Total & 16781,869 & 14051 & & & \\
\hline
\end{tabular}

VI: Centro (Grupo1: E. P. S. de Jaén, Grupo 2: E. U. Politécnica de Linares, Grupo 3: E. U. Trabajo Social, Grupo 4: Facultad Ciencias Experimentales, Grupo 5: Facultad Ciencias Sociales y Jurídicas, Grupo 6: Facultad Humanidades y CCEE, Grupo 7: E.U. Enfermería).

VD: Dimensión 1, Dimensión 2, Dimensión 3, Dimensión 4, Valoración General.

(*) Diferencias significativas a nivel 0.05 .

\section{Conclusiones}

En relación con los resultados del estudio descriptivo de la investigación hemos constatado que los estudiantes de la Universidad de Jaén valoran positivamente la docencia. Ésto queda de manifiesto en la puntuaciones obtenidas de las valoraciones de los encuestados, en donde en todas las variables las puntuaciones medias son superiores al valor central de la escala de respuesta, es decir son superiores a 3 (recordemos que las alternativas de respuesta están comprendidas entre 1 y 5 puntos) para el total de la muestra. Es preciso detenernos en analizar con más detalle este aspecto, ya que el $83,8 \%$ de la muestra asigna una valoración superior a 3 puntos a la valoración global, lo que nos indica que la valoración es positiva.

Así ocurre en la dimensión Obligaciones Docentes-evaluación en donde el 56,7\% de los encuestados otorgaron al Cumplimiento del horario por el docente la máxima puntuación posible. Las altas valoraciones hacen que está dimensión sea la mejor valorada, seguida de 
la Metodología, Medios y Recursos, y en ultimo lugar, la Interacción con el alumnado. No podemos olvidar que todas las dimensiones analizadas tienen una valoración media superior a 3,5 puntos.

En estudios precedentes (Molero 2004a, 2004b, Molero y Ruiz, 2003) hemos obtenido evidencias que nos indican que la dimensión Interacción con el alumnado, a pesar de ser la menos valorada, es la que más predice la valoración de la docencia al realizar un estudio de regresión entre las diferentes dimensiones (predictoras o independientes) y la satisfacción con la docencia (criterio o dependiente).

En relación con los análisis de la varianza realizados destacamos la existencia de diferencias significativas a nivel estadístico entre el sexo y la valoración de la docencia en todas las dimensiones que la componen y en su conjunto.

En el segundo de los análisis de la varianza realizado hemos comprobado la existencia de diferencias significativas, a nivel estadístico, entre los distintos centros o facultades de la Universidad de Jaén y la valoración de la docencia. Destacamos que la Escuela Politécnica Superior de Jaén y la Facultad de Humanidades y C.C.E.E. presentan diferencias significativas, a nivel estadístico, con todos los restantes centros o facultades analizados. Entre el resto de los grupos existen estas diferencias en la gran mayoría de los casos. Por lo que podemos afirmar que cada centro o facultad tiene unas características particulares en su enseñanza y aprendizaje que los diferencian de los demás.

Consideramos necesario detallar un poco más estos dos análisis, por tanto hemos realizado un análisis combinado de los dos con el fin de conocer en que facultades o centros existen diferencias significativas en las valoraciones realizadas en función del sexo del alumnado encuestado. En relación a las mujeres, destacamos la aparición de diferencias significativas entre las estudiantes de la Escuela Politécnica Superior y la Facultad de Humanidades y C.C.E.E. con las demás encuestadas en todas las facultades y todos los centros. En el polo opuesto estarían las estudiantes de las Escuela Universitaria de Enfermería las cuales solo presentan diferencias significativas con E. Politécnica Superior, Facultad de Humanidades y C.C.E.E. (como hemos comentado) y con la Facultad de Ciencias Experimentales.

Los hombres encuestados tienen unas valoraciones distintas, aunque hay algunas semejanzas con las mujeres. Los centros o facultades que presentan mayores diferencias entre los encuestados (hombres) son la Facultad de Humanidades y Ciencias de la Educación (diferencias significativas con E. Politécnica Superior de Jaén, E.U. Politécnica de Linares y Facultad de Ciencias Experimentales) y la Facultad de Ciencias Sociales y Jurídicas (diferencias significativas con E. Politécnica Superior de Jaén, E.U. Politécnica de Linares y Facultad de Ciencias Experimentales). Según los datos que presentamos vemos como las valoraciones del alumnado de una misma Facultad/Centro varían en función de su sexo, aunque hay coincidencias como es el caso de la Facultad de Humanidades y Ciencias de la Educación, siendo las más valoradas y las que presentan más diferencias con el resto.

Una vez aprobados los reales decretos (R.D. 55/2005 y R.D. 56/2005) que regulan los estudios de Grado y Posgrado, siguiendo la normativa del "Crédito Europeo", hay que prever las vías que se van a emplear para la evaluación de la docencia universitaria. Estas medidas tendrán que contemplar la "nueva forma" de trabajar de los docentes y del alumnado, basadas fundamentalmente en cambios metodológicos (trabajo autónomo alumnado, activi- 
dades supervisadas, tutorías colectivas, tutorías virtuales, actividades autodirigidas, enseñanza telemática, etc.), con la finalidad de poder llevar a cabo la evaluación de los docentes universitarios.

Estos nuevos ámbitos exigen el uso de diferentes fuentes de información, además de los cuestionarios de opinión que tradicionalmente viene cumplimentando el alumnado. Junto a la opinión de los estudiantes, es necesaria la aportación de evidencias por parte de los propios docentes y de los responsables de Centros, titulaciones y departamentos. Otra fuente importante de información en los procesos de evaluación de la docencia, a veces olvidada, es la valoración de los egresados sobre los procesos formativos y su utilidad para la inserción laboral.

No cabe duda que la diversidad de fuentes y evidencias en los procesos de evaluación de la docencia, nos van a aportar datos fundamentales, tanto para la mejora de los procesos de enseñanza-aprendizaje como de la necesaria orientación que hay que realizar durante estos procesos.

\section{Referencias Bibliográficas}

Abalde, E., Salvador de, X., González, R. y Muñoz, J. M. (1995). “Análisis de la docencia universitaria por los alumnos de la universidad de La Coruña (1993-94)". En AIDIPE, Estudios de investigación educativa en intervención psicopedagógica (pp. 289-292). Valencia: AIDIPE.

Abrami, P. C., Cohen, P. A. y d'Apollonia (1990). "Validity of Student Ratings of Instruction: GAT we know and what do not". Journal of Educational Psychology, 82 (2), 219-231.

Aleamoni, L. M. (1981). "Student Ratings of Instruction". En J. Millman (Eds.), Handbook of Teacher Evaluation. Beverly Hills, CA: Sage.

Aparicio, J. J., Tejedor, F. J. y Sanmartín, R. (1982). La enseñanza universitaria vista por los alumnos: Un estudio para la evaluación de los cursos en la enseñanza superior. Universidad Autónoma de Madrid. Instituto de Ciencias de la Educación.

Apodaca, P. y Grad, H. (2002). "Análisis dimensional de las opiniones de los alumnos universitarios sobre sus profesores: Comparación entre técnicas paramétricas y no paramétricas". Revista de Investigación Educativa, 20 (2), 385-409.

Barr, A. S. (1948). "The measurement and prediction of teaching efficiency: A summary of investigations". Journal of Experimental Education, 16, 203-283.

Buendía, L. (1994). "El proceso de investigación”. En M. P. Colás y L. Buendía, Investigación educativa (pp. 69-108). Sevilla: Alfar.

Cajide, J. (1994). “Análisis factorial confirmatorio de las características de la calidad docente universitaria (solución LISREL)”. Bordón, 46(4), 389-405.

Cajide, J., Doval, L. y Porto, A. (1996). Perspectivas actuais da avaliación da docencia universitaria. Santiago de Compostela: Universidad de Santiago.

Centra, J. A. (1988). Determining Faculty Effectiveness. San Francisco-Londres: Jossey-Bass.

Centra, J. A. (1993). Reflective Faculty Enhacing teaching and determining Faculty Effectiveness The Jossey-Bass Higher and Adult Education series. San Francisco: Jossey-Bass.

Centra, J. A. (2000). "Evaluating the teaching Portfolio: A Role for colleagues". New Directions for teachings and learning, 83, 87-93. 
Consejo de Coordinación Universitaria (2002). Informe Global. Plan Nacional de Evaluación de la Calidad de las Universidades (1996-2000). Documento descargado de Internet el 15 de marzo de 2003 de: www.mec.es/consejou/index.html.

Cook, S. S. (1989). "Improving the quality of student ratings of instruction: A look at two strategies". Research in Higher Education, 30 (1), 31-45.

Creager, J. A. (1950). "A multiple-factor analysis of the Purdue rating scale for instructors?". Purdue University Studies in Higher Education, 70, 75-96.

Doyle, K. O. (1983). "Evaluating Teaching”. Innovation Abstracts, 5 (27).

Drucker, A. J. y Remmers, H. H. (1950). "Do Alumni and Students Differ in Their Attitudes Towards Instructors?". Purdue University Studies in Higher Education, 70, 62-64.

Escudero, T. (1989). "Aproximación pragmática a la evaluación de la universidad”. Revista de Investigación Educativa, 7 (13), 93-12.

Escudero, T. (2002). "Evaluación institucional: algunos fundamentos y razones". En V. Álvarez y A. Lázaro (Coords.), Calidad de las universidades y orientación universitaria (103-138). Archidona (Málaga): Aljibe.

García Ramos, J. M. (1989). "Evaluación de la eficacia docente”. En R. Pérez Juste y J. M. García Ramos, Diagnóstico, evaluación y toma de decisiones (cap. 22). Madrid: Rialp.

García Ramos, J. M. (1997). "Análisis factorial confirmatorio en la validación del constructo competencia docente del profesor universitario". Bordón, 49 (4), 361-391.

García Ramos, J. M. (1999). "Análisis multirasgo-multimétodo en la validación de instrumentos para la evaluación de la calidad docente en instituciones universitarias". Revista Española de Pedagogía, 57 (214), 417-444.

García-Valcárcel, A. (1992). "Características del buen docente universitario según estudiantes y profesores". Revista de Investigación educativa, 19, 31-50.

Gavira, J. L. (2000). “Cambios en las técnicas cuantitativas de investigación socio-educativa”. En Sociedad Española de Pedagogía (Ed.), Actas del XII Congreso Nacional y I Iberoamericano de Pedagogía. Tomo I, Ponencias (pp. 39-86). Madrid: S.E.P.

González Such, J., Jornet, J. M., Pérez, A. y Ferrández, M. R. (1995). "Factores intervinientes en la valoración del profesor por parte del estudiante". Documento presentado en VI Seminario sobre Modelos de Investigación Educativa, Madrid, 23-25 de septiembre de 1995.

González, J. (1997). Estudio de un instrumento para la evaluación del profesorado universitario. Tesis doctoral. Universitat de València.

Good, T. L. y Mulryan, C. (1990). "Teacher ratings: A call for teacher control and self-evaluation". En J. Millman y L. Darling-Hammond (Eds.), The New Handbook of Teacher Evaluation (pp. 191215). Newbury park, Cal: Sage Publications.

Guthrie, E. R. (1954). The Evaluation of Teaching: A Progress Report. Seatle: University of Washington.

Jiménez, C. (1985). "La eficacia docente de la UNED: aproximación a un modelo". Revista de Investigación Educativa, 3 (6), 401-406.

Jornet, J. M., Suárez, J. M., González Such, J. y Pérez, A. (1996). "Evaluación de la actividad universitaria". En G. Quintas (Ed.), Reforma y Evaluación de la universidad (pp. 189-244). Valencia: Servei de Publicacions de la Universitat de València.

Jornet, J. M., Villanueva, P., Suárez, J. M. y Alfaro, I. J. (1987). "Proyecto de implantación de un sistema de evaluación del profesorado en la Universitat de València". En Universitat de València (Ed.), Consideraciones metodológicas sobre Evaluación y Mejora de la Docencia Universitaria. València: Servei de Formació Permanent, Universitat de València. 
Marsh, H. W. (1987). "Student's evaluation of university teaching: research findings. Methodological issues and directions for future research". International Journal of Educational Research, 11(3), 253-288.

Marsh, H. W. y Roche, L. A. (1992). "The use of Student Evaluations of University Teaching in Different Settings: The Applicability Paradigm". Australian Journal of Education, 36 (3), 278-300.

Marsh, H. W. y Roche, L. A. (1993). "The use of student's Evaluation and Individually Structured Intervention to Enhance University Teaching Effectiveness". American Educational Research Journal, 30 (1), 217-251.

Marsh, H. W., Touron, J. y Wheeler, B. (1985). "Student's evaluations of university instructors: The applicability of American instruments in a spanish setting". Teaching and teacher Education, 1 (2), 123-138.

Mateo, J. (1987). "La evaluación del profesorado universitario. Algunas reflexiones en torno al estado de la cuestión". En Consideraciones metodológicas sobre la evaluación y mejora de la docencia universitaria (pp. 133-203). Valencia: Servei de Formació Permanent, Universitat de València.

Mateo, J. (2000). "La evaluación del profesorado y la gestión de la calidad de la educación. Hacia un modelo comprensivo de evaluación sistemática de la docencia". Revista de Investigación Educati$v a, 18$ (1), 7-36.

Miguel, M. de (1989). Criterios para la evaluación del profesorado universitario. Oviedo: KRK Ediciones.

Molero, D. (2003). "Ficha de investigación: Construcción de un instrumento para la evaluación de la docencia universitaria". Revista de Investigación Educativa, 21 (2), 553-534.

Molero, D. (2004a). La evaluación de la docencia en la universidad. Jaén: Servicio de Publicaciones de la Universidad de Jaén.

Molero, D. (2004b). Construcción de un instrumento para la evaluación de la docencia universitaria. Tesis doctoral. Jaén: Servicio de Publicaciones de la Universidad de Jaén. Formato CD.

Molero, D. y Ruiz, J. (2003). "Validación del cuestionario de evaluación de la docencia universitaria (CEDU)". En L. Buendía, T. Pozo, D. González y C. Sánchez, Investigación y sociedad (pp. 1021-1027). Granada: Grupo Editorial Universitario, AIDIPE.

Muñoz, J. M., Ríos, M. P. y Abalde, E. (2002). "Evaluación docente vs. Evaluación de la calidad". Revista Electrónica de Investigación Educativa, 8 (2), 103-134.

Muñoz, J. M., Ríos, M. P. y Abalde, E. (2003). "Evaluación docente vs. Evaluación de la calidad”. En L. Buendía, T. Pozo, D. González y C. Sánchez, Investigación y sociedad (pp.863-868). Granada: Grupo Editorial Universitario, AIDIPE.

Real Decreto 1947/1995, de 1 de diciembre, por el que se establece el Plan Nacional de Evaluación de la Calidad de las Universidades (BOE, 9 de diciembre de 1995).

Real Decreto 408/2001, de 20 de abril, por el que se establece el II Plan de la Calidad de las Universidades (BOE, 21 de abril de 2001).

Real Decreto 55/2005, de 21 de enero, por el que se establece la estructura de las enseñanzas universitarias y se regulan los estudios universitarios de grado (BOE, 25 de enero de 2005).

Real Decreto 56/2005, de 21 de enero, por el que se regulan los estudios universitarios de posgrado (BOE, 25 de enero de 2005).

Remmers, H. H. (1931). "The equivalence of judgements and test items on the sense of the SpearmanBrown formula". Journal of Educational Psychology, 22, 66-71.

Remmers, H. H. (1934). "Reliability and halo effect on high school and college student's judgement of their teachers". Journal of Applied Psychology, 18, 619-630. 
Remmers, H. H., Martin, F. D. y Elliot, D. N. (1949). "Are student ratings of their instructors related to their grades?". Purdue University Studies in Higher Education, 44, 17-26.

Rodríguez Espinar, S. (1987). "La evaluación del profesorado universitario. Análisis de la opinión del profesorado universitario de la Universidad de Barcelona". En Consideraciones Metodológicas sobre la Evaluación y Mejora de la Docencia Universitaria. Valencia: Servei de Formacio Permanent de la Universitat de Valencia.

Rodríguez Gómez, G. (2000). "La evaluación de la actividad docente en la universidad: entre el sueño y la realidad". Revista de Investigación Educativa, 18 (2), 417-432.

Ruiz, J. (2000). "La evaluación de la enseñanza por los alumnos en el Plan Nacional de Evaluación de la Calidad. Construcción de un instrumento de evaluación". Revista de Investigación Educativa, 18 (2), 443-456.

Tejedor, F. J. (1985). "Problemática de la enseñanza universitaria". Revista de Investigación Educati$v a, 3$ (6).

Tejedor, F. J. (1986). Evaluación del profesorado de la Universidad de Santiago por los alumnos (curso 85-86). Santiago de Compostela: Universidad de Santiago de Compostela.

Tejedor, F. J. (2003). "Un modelo de evaluación del profesorado universitario". Revista de Investigación Educativa, 21 (1), 157-182.

Tejedor, F. J. y García-Valcarcel, A. (1996). "La evaluación de la calidad de la docencia universitaria (en el marco de la evaluación institucional) desde la perspectiva del alumno". En F. J. Tejedor y J. L. Rodríguez (Coords.), Evaluación educativa II. Evaluación institucional (pp. 93-122). Salamanca: Universidad de Salamanca.

Tejedor, F. J., Ausín, T., Garcia-Varcálcel, A., Herrera, E., Martín, J. F., Nieto, S., Rodríguez, M. J. y Sánchez, M. C. (1995). "Elaboración de un instrumento de evaluación de las actitudes de los universitarios hacia la Universidad, los alumnos y los profesores". En AIDIPE (Ed.), Estudios de Investigación Educativa en Intervención Psicopedagógica. Valencia: AIDIPE.

Tejedor, F. J., Jato, E. y Mínguez, C. (1988). "Evaluación del profesorado universitario por los alumnos de la Universidad de Santiago". Studia Paedagogica, 20, 73-134.

Tierno, J. Ma . y Jiménez, B. (2003). "La opinión de los responsables universitarios sobre la valoración de la docencia por parte de los alumnos”. En L. Buendía, T. Pozo, D. González y C. Sánchez, Investigación y sociedad (pp. 1147-1153). Granada: Grupo Editorial Universitario, AIDIPE.

Universidad de Jaén (2002). Encuesta de evaluación docente. Tipología de informes. Universidad de Jaén, Gabinete de Calidad.

Wilson, W. R. (1999). "Students Rating Teacher". Journal of Higher Education, 70 (5), 562-571.

Wolf, R. M. (1990). Evaluation in Education. Nueva York: Praeger.

Fecha de recepción: 11-12-04

Fecha de revisión: 6-10-05

Fecha de aceptación: 19-10-05 


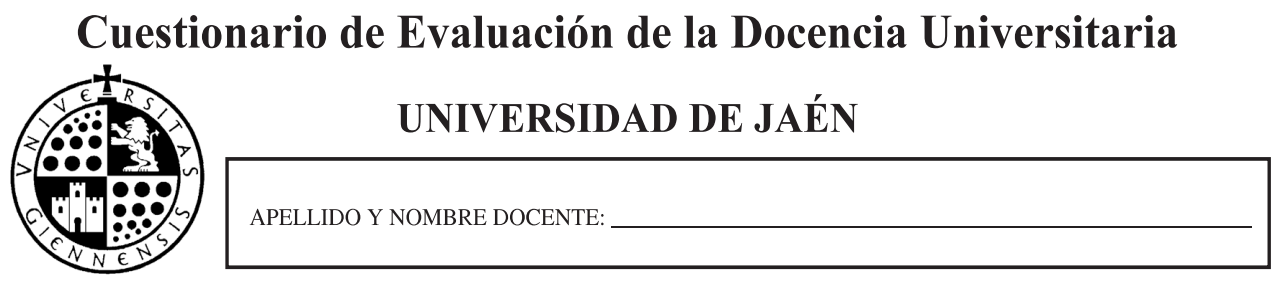

DATOS DE LA ASIGNATURA EVALUADA:

Asignatura: Titulación:

Curso: Grupo: T eoría / Prácticas:

\section{SI ESTÁ COMPLETAMENTE DE ACUERDO CON LAS AFIRMACIONES MARQUE UN 5 SI ESTÁ EN COMPLETO DESACUERDO MARQUE UN 1 \\ REALICE LA VALORACIÓN UTILIZANDO LOS PUNTOS MEDIOS DE LA ESCALA PROCURE CONTESTAR A TODOS LOS ÍTEMS}

1. El profesor ha cumplido el horario de clase

2. El profesor atiende correctamente al alumnado en horas de tutorías

3. El programa se define con claridad (metodología y contenidos)

4. El profesor anticipó los objetivos del curso y de cada tema

5. El método de evaluación del profesor ha sido conocido con antelación a la fecha de examen

6. E profesor tiene en cuenta la opinión del alumnado en la marcha de la asignatura

7. El profesor muestra interés por que el alumnado aprenda

8. El profesor motivó y facilito la participación del alumnado en clase

9. Existe una buena relación entre el profesor y el alumnado

10. El profesor ha contribuido a que me guste la asignatura

11. El profesor ha contribuido a que comprenda la importancia de la asignatura

12. El profesor imparte sus clases con claridad, organización y coherencia

13. El profesor responde con exactitud y precisión a las preguntas que se le hacen

14. El profesor domina la asignatura que imparte

15. La metodología de enseñanza utilizada resulta adecuada a las características del grupo y de la asignatura

16. Los problemas, ejemplos o prácticas que plantea están bien pensados para el contexto de la asignatura

17. El material técnico y de laboratorio necesario para la esta asignatura es el apropiado

18. La bibliografía y el material didáctico recomendado resulta útil para preparar la asignatura

19. Sus clases están bien preparadas

20. Existe coordinación entre la parte teórica y la práctica de la asignatura

21. El profesor ha cumplido el programa de la asignatura planteado al inicio del curso

22. Los exámenes / evaluaciones que se han realizado se ajustan a los objetivos y a los contenidos trabajados en clase

23. El profesor respeta los criterios de evaluación establecidos en la asignatura

24. El sistema de evaluación de la asignatura permite la revisión por parte del alumnado

25. Estoy satisfecho, en general, con respecto al profesor de la asignatura

\section{LA PROPIA ACTUACIÓN COMO ESTUDIANTE}

26. Sexo

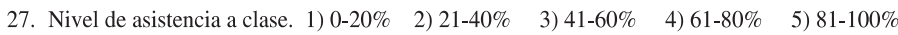

28. El planteamiento docente de la asignatura fomenta el estudio y el trabajo personal

29. He podido acceder en la biblioteca, a la bibliografía y lecturas recomendadas por el profesor
30. Ha utilizado la tutoría
1) $\mathrm{Si}$
2) No

31. Nivel de satisfacción con la tutoría

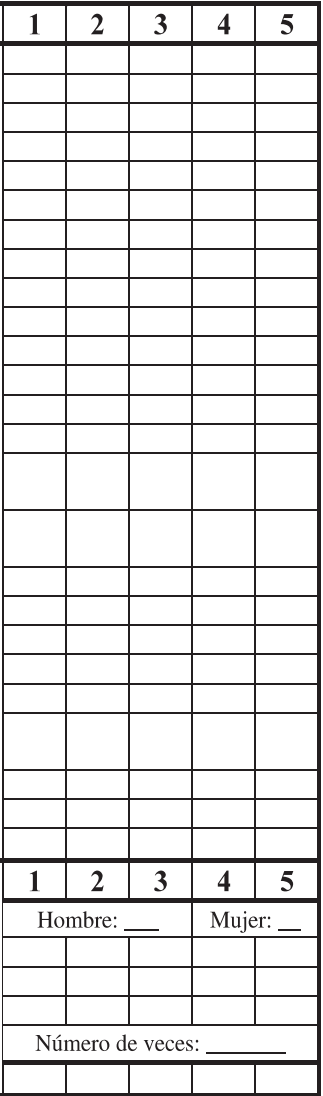

